Classification

Physics Abstracts

61.14.Dc-61.16.-d

\title{
Some Practical Problems when Using the Direct Potential Method in HREM Image Simulation
}

\author{
Dong Tang $\left({ }^{*}\right)$ and Dominique Dorignac \\ CNRS, CEMES-LOE, MEA, BP 4347, 31055 Toulouse, France
}

(Received January 30; accepted March 11, 1996)

\begin{abstract}
Résumé. - On étudie l'influence de l'emploi de la "méthode du potentiel direct", méthode que nous avons récemment proposée pour déterminer le potentiel projeté d'une mince couche de matière, sur le calcul de la fonction d'onde électronique à la sortie d'un objet cristallin effectué à l'aide de la procédure multicouche. On examine en particulier la façon la plus efficace d'utiliser les paramètres de calcul les plus significatifs. On donne également une explication simple et probable de l'allure des courbes Pendellösung multi-faisceaux.
\end{abstract}

\begin{abstract}
The effects on the exit wave function computation using the multislice method after calculating the slice projected potential by our newly proposed "direct potential method" are systematically studied. In particular, the most efficient use of all the relevant computing parameters is discussed. A possible and simple explanation of many-beam Pendellösung curves is also given.
\end{abstract}

\section{Introduction}

We recently proposed a new method of calculating the slice projected potential for multislice (MS) image simulation in high resolution electron microscopy (HREM) [1, 2]. The idea of such a "direct potential method" is very simple. The calculation of the two-dimensional (2D) projected potential for a slice of thickness $\Delta z$ is performed directly in real space, from a 1D integration of the $3 \mathrm{D}$ crystal potential, $\phi(\mathbf{r})$, as

$$
\phi^{\mathrm{P}}(\boldsymbol{\rho})=\frac{1}{\Delta z} \int_{0}^{\Delta z} \phi(\mathbf{r}) \mathrm{d} z,
$$

where $\mathbf{r}=(x, y, z)$ and $\boldsymbol{\rho}=(x, y)$ represent the real space 3D and 2D Cartesian coordinates. Accurate centro-symmetric atomic potentials are used, which are based on the relativistic HartreeFock-Slater model and tabulated with very fine and appropriate radius increments [3]. The calculation is also performed following the high-energy quantum-mechanical quasi-classical approximation, the slice transmitted-wave being expressed as a pure phase modulation of the incident

$\left(^{*}\right)$ Present address: Philips EOBV, Application Laboratory AAE, PO Box 218, 5600 MD Eindhoven, The Netherlands 
electron wave [4]. The validity of this approximation is carefully examined by estimating the electron wave phase shift due to the crystal potential

$$
\chi(\rho) \approx \sigma \Delta z \phi^{\mathrm{P}}(\boldsymbol{\rho})
$$

for which the interaction constant $\sigma$ is defined as $\left(2 \pi m e \lambda / h^{2}\right)$, with $e$ the (absolute) electron charge, $m$ the relativistic mass of the electron, $h$ Planck's constant and $\lambda$ the incident electron wavelength, the phase-shift-change $\Delta \chi$ over a distance of the order of $\lambda$ necessarily being far less than unity

$$
\Delta \chi \ll 1
$$

The direct potential method has several major advantages over the conventional one. Firstly, the direct method is a more accurate way of calculating the projected potential since it replaces the usual discrete numerical Fourier transform (FFT), $\phi^{\mathrm{P}}(\rho)=\bar{F} \tilde{\phi}_{h k 0}$, by the simple finite integral (1) over $\Delta z$, and it also avoids the errors arising from analytical fitting of the first Born atomic scattering factors for electrons, which are needed to calculate the 3D Fourier coefficients of the crystal potential, $\tilde{\phi}_{h k l}=F \phi(\mathbf{r})$, where $F$ and $\bar{F}$ represent finite FFTs from the real to reciprocal space and from the reciprocal to real space, respectively [5-7]. Secondly, it can easily be applied to cases in which the potential distribution along the $z$ dimension is important, such as the calculations involving the high-order Laue diffraction zones [8]. Thirdly, it ensures that the phase-object approximation is valid for a single slice of thickness $\Delta z$, either the crystal repeat distance or an integer division of it: the phase shift $\chi$ thereby obtained being continuous, the criterion (3) can effectively be used to judge the validity of the slice transmission function, which is constructed as $\exp [i \chi(\rho)]$. Such a validity criterion cannot be easily applied with the conventional method because of the discontinuity of the discretely calculated projected potential [2]; and the unitarity test which is generally used in conventional methods, $|\exp [i \chi(\rho)]|^{2}=1$, is more sensitive to the rounding errors created in the numerical calculation than the physical properties of the transmission function itself [9]. Fourthly, the calculation of $\phi^{\mathrm{P}}$ according to the quasi-classical approximation (3) is entirely consistent with the subsequent MS procedure, used to calculate the crystal exit wavefield, since the MS approach is also based on the phase-object approximation [10].

However, since the new direct method introduces more computing parameters, care must be taken to choose correctly those which are relevant in order to make the HREM image simulation as precise as possible. In this paper, the downstream effects on image simulation when using the direct potential method are discussed and ways of dealing with the computing parameters in practice are indicated. Henceforth, for simplicity, the effects associated with the direct calculation of the transmission function are termed "approximation", and the effects rising from the forward MS iteration will be termed "iteration".

\section{Parameters for Computing}

Apart from the total thickness of the crystal, $T$, at least four parameters are important for the exit-wave calculation. They are (not necessarily in order of importance): the maximum acceptable quasi-classical phase-shift-change, $\Delta \chi_{\max }$; the minimum classical impact parameter $\rho_{\min }=\left|\rho_{\min }\right|$, which is related to the value of the projected potential at the centre of the heaviest atomic chain in the structure,

$$
\phi_{\max }^{\mathrm{P}} \equiv \phi^{\mathrm{P}}\left(\rho_{\min }\right) ;
$$

the slice thickness, $\Delta z$; and the number of pixels for sampling in real space (or in reciprocal space for the Fourier space size $),(M \times N)$.

The parameters $T$ and $\Delta z$ are familiar to conventional program users. Many authors have already outlined how to obtain the best values of them following the unitarity and normalization 


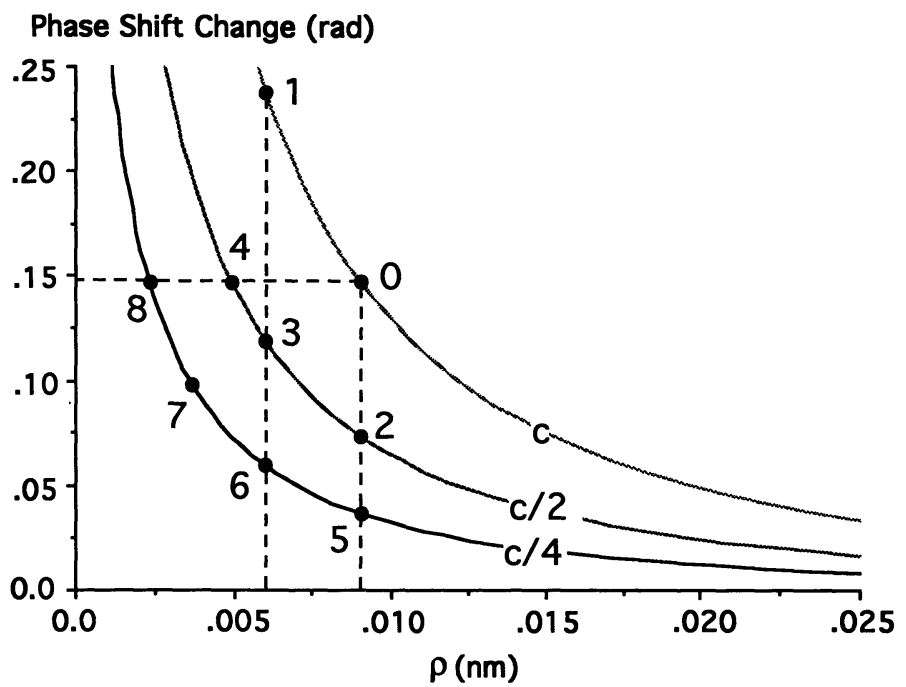

Fig. 1. - The curves of the phase-shift-change $\Delta \chi$ suffered by a quasi-classical $300 \mathrm{keV}$ electron wave scattered by an atomic chain in a copper crystal along a $\langle 011\rangle$ direction versus the classical electron impact parameter $\rho$, for different slice thicknesses: $\Delta z=c, c / 2$ and $c / 4$. The numbered black dots represent different calculation tactics (\#) which will be mentioned later.

tests (see for example [11]). The two new parameters, $\Delta \chi_{\max }$ and $\rho_{\min }$, are actually not independent, because as long as $\Delta \chi_{\max }$ is fixed, the $\rho_{\min }$ value is also necessarily restricted with a lower limit, which is set up by a smaller sampling distance, $\rho_{\text {ssd }}$. For instance, for a monoclinic projected unit cell, having the crystal constants $a, b$ and $c$,

$$
\rho_{\min }<\rho_{\text {ssd }}=\min (\Delta x=a / M, \Delta y=b / N) .
$$

The following computer tests correspond to this case: they have been performed with electrons of $300 \mathrm{keV}$ kinetic energy $(\lambda=1.969 \mathrm{pm})$ for a crystal of copper $(\mathrm{Cu}: Z=29)$ observed along the $\langle 011\rangle$ direction, for which $a=0.3615 \mathrm{~nm}, b=0.2556 \mathrm{~nm}$ and $c=0.5112 \mathrm{~nm}$ along the virtual [001] incidence if we refer to the projected cell.

2.1 The Maximum Quasi-Classical Phase-Shift-Change $\Delta \chi_{\max }$ - - The first parameter to be considered is the acceptable maximum value of the quasi-classical phase-shift-change over a wavelength. Apart from the loose relationship (3), there is no rigorous theoretical definition to show how much smaller than unity this value should be. We have therefore adopted the value

$$
\Delta \chi_{\max } \approx 0.15 \mathrm{rad}
$$

for the following tests.

Figure 1 shows the $\Delta \chi(\rho)$ curves corresponding to three different slice thicknesses. In the chosen $\langle 011\rangle$ projected direction, there is one $\mathrm{Cu}$ atom on top of another in one unit cell, which makes a two-atom atomic chain in the $z$ direction centred on $\rho=0$. According to the different values of $\Delta z$, there are two $\mathrm{Cu}$ atoms in the "atomic chain" if $\Delta z=c$, but only one atom (or half an atom) if $\Delta z=c / 2$ (or $\Delta z=c / 4$ ) such that the phase shift $\chi$ and its change $\Delta \chi$ in the two later cases are also halved (or quartered). The horizontal line corresponds to $\Delta \chi=0.1479 \mathrm{rad}$, close to the maximum phase-shift-change (6), which is tolerable. The numerical labels in the figure refer to the various calculation tactics (\#), which will be discussed later. 


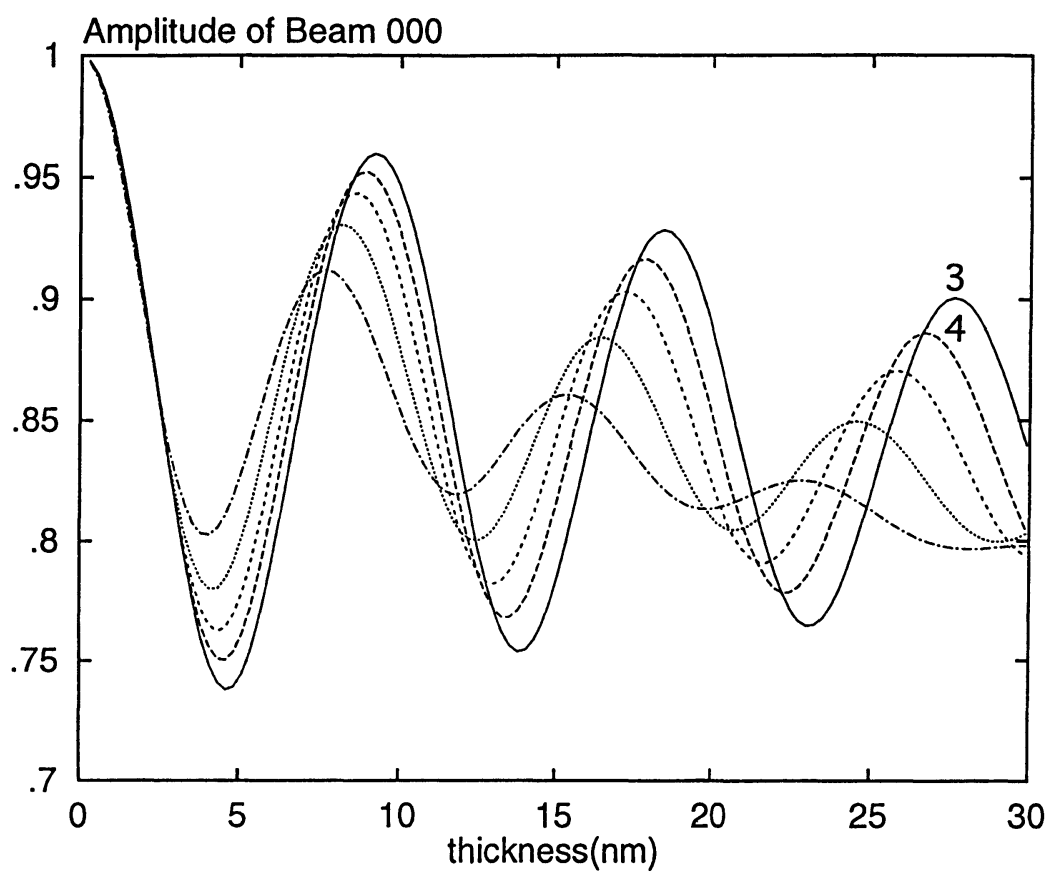

Fig. 2. - The dynamical amplitude of beam (000) for a $300 \mathrm{keV}$ electron wave, down the $\langle 011\rangle$ direction of a copper crystal, against the crossed thickness $T$, calculated for various minimum impact parameters $\rho_{\min }$ : $6,4.9,4,3$ and $2 \mathrm{pm}$, corresponding respectively to the order of decreasing oscillation amplitude. The other fixed computing parameters are slice thickness $\Delta z=c / 2$ and Fourier space size $(M \times N)=32 \times 32$. The numbers marked in the figure correspond to the tactics \#3 and \#4 of Figure 1.

2.2 The Minimum Classical Impact Parameter $\rho_{\min }$. - In order to represent the projected potential accurately at the centre of an atomic chain by $\phi^{\mathbf{P}}\left(\rho_{\min }\right)$, according to (4), in place of $\phi^{\mathrm{P}}(\rho \rightarrow 0)$ which theoretically tends to infinity, it is important to choose a correct $\rho_{\min }$ value.

Figure 2 shows the amplitude-thickness (Pendellösung) curves computed for the transmitted beam $(h=0, k=0, l=0)$, with $\Delta z=c / 2$ and $(M \times N)=(32 \times 32)$, for various minimum impact parameters: $\rho_{\min }=6$ (the highest solid line), 4.9 (the second highest), 4 (the middle), 3 (the second lowest) and $2 \mathrm{pm}$ (the lowest line), respectively. The first two curves correspond to the tactics \#3 and \#4 in Figure 1. The diffracted beams are not represented here, since they display a very similar behaviour (see Sect. 2.5 and Fig. 6). It is clear from the figure that reducing the value of $\rho_{\min }$ will reduce both the period $(P)$ and the amplitude $(A)$ of the oscillations of the Pendellösung curves. It seems that there is a virtual damping envelope function on the top of the Pendellösung curves: the smaller $\rho_{\min }$ is, the more severe is the damping. Further calculations with bigger or smaller $\rho_{\min }$ resulted in a similar and predictable behaviour.

The Pendellösung curves are known to be the result of the interference between many Bloch waves $\left({ }^{1}\right)$ having slightly different wavevectors [12]. Since the anomalous absorption is different for each Bloch wave, the amplitude $A$ of the oscillations is expected to decrease as enough there were a damping effect. The most simple example is a two-beam case: the anomalous absorption for

$\left({ }^{1}\right)$ We note in passing that it would be inappropriate to compare the direct method with the Bloch-wave procedure, since the latter does not invoke the notion of projected potential, but only needs the calculation of the Fourier coefficients of the potential (see [15]). Because of this fundamental difference, the above comparison is out of the scope of this paper. 
one Bloch wave is stronger, the interference is more and more dominated by another Bloch wave and the beating between these two Bloch waves is reduced in intensity. In the test the anomalous absorption is not considered at all, apart from a virtual aperture, and we can thus suppose that the most reliable curve should have the smallest damping. On the other hand, a larger $\rho_{\min }$ leads to a smaller $\Delta \chi_{\max }$, from the "approximation" point of view, and to a relatively smaller $\phi^{\mathrm{P}}\left(\rho_{\min }\right)$, from the dynamical scattering point of view: that is, in both views, to a better approximation for single-slice scattering and hence to a more reliable calculation. When $\rho_{\min }=6$ and $4.9 \mathrm{pm}, \Delta \chi_{\max }$ is about 0.121 and $0.148 \mathrm{rad}$ respectively, while when $\rho_{\min }$ is smaller $\Delta \chi_{\max }$ exceeds the chosen criterion of about $0.15 \mathrm{rad}$. As clearly shown in Figure 2, the choice of an extra small $\rho_{\min }$ (i.e. an extra large $\Delta \chi_{\max }$ ) leads apparently to more damping in the Pendellösung curves. The effect can also be considered by noting that the extra high $\phi^{\mathrm{P}}\left(\rho_{\min }\right)$ makes the scattering angles larger and so the energy in a given $(M \times N)$ Fourier space, i.e. in a given diffraction plane area, is lower. This is confirmed by examining the corresponding normalization factors: as an example, for $T=30 \mathrm{~nm}$, the total intensities in the diffraction plane (for an incident wave of amplitude unity) are found to be $I_{\text {dif }}=0.84,0.81,0.79,0.76$ and 0.72 , in the order of increasing damping. Therefore, in Figure 2, the solid curve (\#3), which corresponds to both the smallest damping and the smallest $\Delta \chi_{\max }$, is believed to be the most reliable of all.

However, according to (5) we cannot increase the value of $\rho_{\min }$ indefinitely, as required by the above analysis. This value must be smaller than $\rho_{\text {ssd }}$, the smaller-sampling-distance in two dimensions. Otherwise, the calculated projected potential curve will deviate from the shape of a peak to form either a central platform or, even worse, a central valley. To consider the validity of the "approximation" and, at the same time, to represent the projected potential with as little distortion as possible, a rough criterion that the ratio

$$
R=\phi^{\mathrm{P}}\left(\rho_{\text {ssd }}\right) / \phi^{\mathrm{P}}\left(\rho_{\min }\right) \approx 80 \%
$$

is proposed. It will be used for the following calculation. Both tactics corresponding to points \#3 and \#4 in Figure 1 are in this region.

2.3 The Slice Thickness $\Delta z$. - The principle of the MS iteration is that if $\Delta z$ tends to zero the effects of scattering and propagation of the electron wave can be treated separately [10]. In practical work we always deal with a very thin but finite $\Delta z$, i.e. $\Delta z \ll d^{2} / \lambda$ if one is interested in details of size $d$, the point resolution of the microscope.

Figure 3 shows two groups of the Pendellösung curves obtained for three different $\Delta z$ by tactics $\# 0, \# 2$ and \#5 $\left(M \times N=32 \times 16 ; \rho_{\min }=9 \mathrm{pm}\right)$ and by tactics \#1, \#3 and \#6 $(M \times N=$ $\left.32 \times 32 ; \rho_{\min }=6 \mathrm{pm}\right)$. The overall effect of finite slice thickness is clearly reflected by more damping as $\Delta z$ increases, with no other changes, notably a fixed oscillation period $P$. The curves \#5 and \#6 corresponding to $\Delta z=c / 4$ show the highest amplitude $A$ in the individual groups; on the contrary, the curves \#0 and \#1 corresponding to $\Delta z=c$ show the smallest $A$. When considering all the six curves, it is clear that the largest relative damping occurs for the curve \#1, which corresponds to the largest phase-shift-change, $\Delta \chi=0.24 \mathrm{rad}$, as shown in Figure 1. From the point of view of the "approximation", curve \#5 is better than curve \#2, which is in turn better than curve \#0. An identical relationship remains for the group of curves \#6, \#3 and \#1.

The intensity normalization factors for $T=30 \mathrm{~nm}$ give $I_{\text {dif }}=0.90,0.83$ and 0.76 for curves \#6, \#3 and \#1 and $I_{\text {dif }}=0.89,0.81$ and 0.73 for curves \#5, \#2 and \#0, respectively. This confirms the reliability of the calculations and also the fact that the damping observed in the Pendellösung curves computed without absorption is an effect of imprecision. This latter point is also consistent with the conclusion made in the last section.

The differences between the above two groups of curves observed for the oscillation periods $P$ will be discussed in 2.5 . 


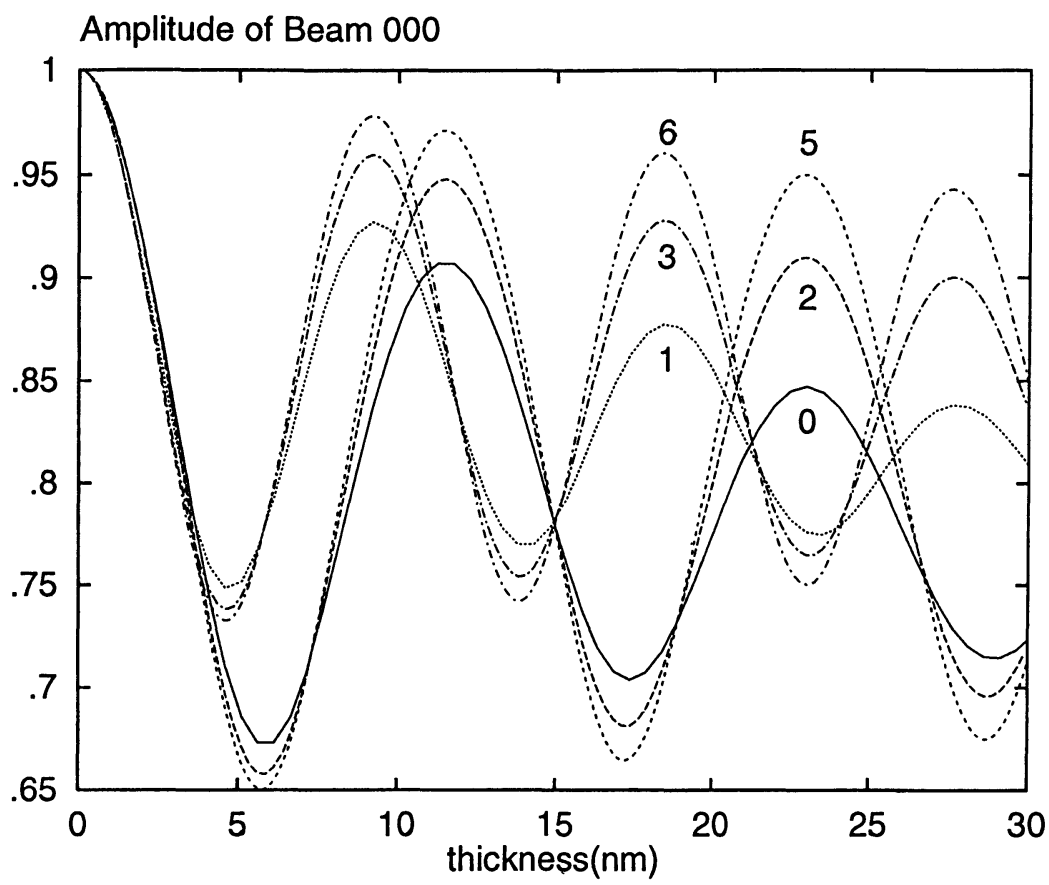

Fig. 3. - The amplitude-thickness curves of beam (000) which show the effect of using different values of $\Delta z$ in multislice calculations. The only difference for curves \#0, \#2 and \#5 (calculated with $M \times N=$ $32 \times 32)$ or for curves \#1, \#3 and \#6 $(M \times N=32 \times 16)$ is $\Delta z=c, c / 2$ or $c / 4$, respectively.

2.4 The Fourier Space Size $(M \times N)$. - This section concerns more the "iteration" than the "approximation" point of view, because if the MS calculation is not needed afterwards, the calculation of the projected potential via the direct potential method has nothing to do with the $(M \times N)$ sampling numbers and the Fourier space size.

Because of the rounding errors, it is preferable to use a Fourier size as large as possible during the MS iteration. However this is limited by the computer memory available and the computing time [11].

Figure 4 shows four Pendellösung curves obtained with $\Delta z=c / 4, \rho_{\min }=2.3 \mathrm{pm}$ and $(M \times N)=$ $(32 \times 16),(32 \times 32),(64 \times 32)$ and $(64 \times 64)$. The last one corresponds to tactic \#8 in Figure 1 . Since a fixed $\rho_{\min }$ is used for all the Fourier sizes, the ratio $R$ changes dramatically: it decreases from $R=75.3 \%$ for $(64 \times 64)$ to $60.6 \%$ for $(64 \times 32)$, to $47.0 \%$ for $(32 \times 32)$ and to $34.8 \%$ for $(32 \times 16)$, respectively. Although the constant value of $\Delta \chi_{\max }=0.148 \mathrm{rad}$ is permitted, an over-damping occurs for those curves corresponding to too small a value of $R$, as discussed in 2.2. Clearly, the increase of $(M \times N)$, with $\rho_{\min }$ remaining unchanged, leads to shorter $P$ and to lower damping.

2.5 The Valid Tactics (\#) and the thickness uncertainty. - In Figure 1, let us consider the rectangle formed by a horizontal line drawn at the level of a chosen $\Delta \chi_{\max }$, two vertical lines plotted symmetrically on both sides of a chosen valid $\rho_{\min }$ with a tolerable distance, and the horizontal axis: all the intersections of the $\Delta \chi(\rho)$ curves within this rectangle will represent valid calculations, termed as valid tactics (\#). If we think, from (7), that a constant ratio $R \approx 80 \%$ is reasonable, different $(M \times N)$ sizes will lead to different valid tactics. 


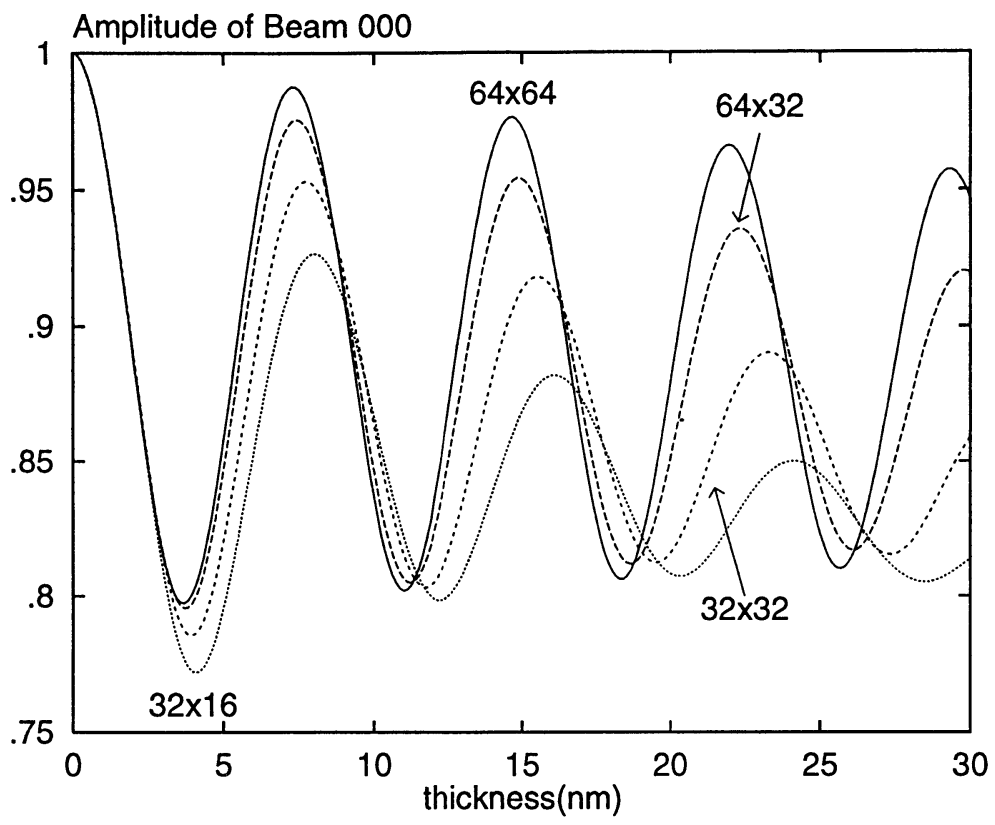

Fig. 4. - The amplitude-thickness curves of beam (000) showing the influence of $(M \times N)$ in the calculation, the values $\rho_{\min }=2.3 \mathrm{pm}$ and $\Delta z=c / 2$ being used for all. The curve for $(M \times N)=64 \times 64$ corresponds to tactic \#8.

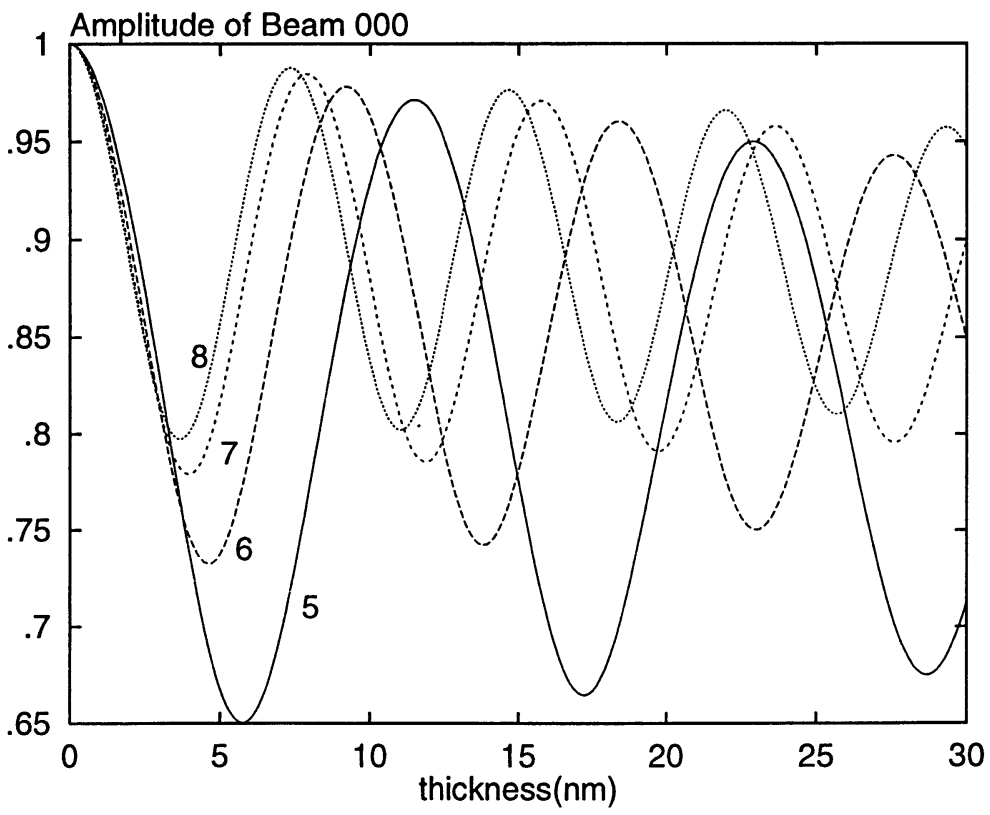

Fig. 5. - The amplitude-thickness curves of beam (000) which show the differences between so called valid calculations with $\Delta z=c / 4$ (tactics \#5, \#6, \#7 and \#8 of Fig. 1). 


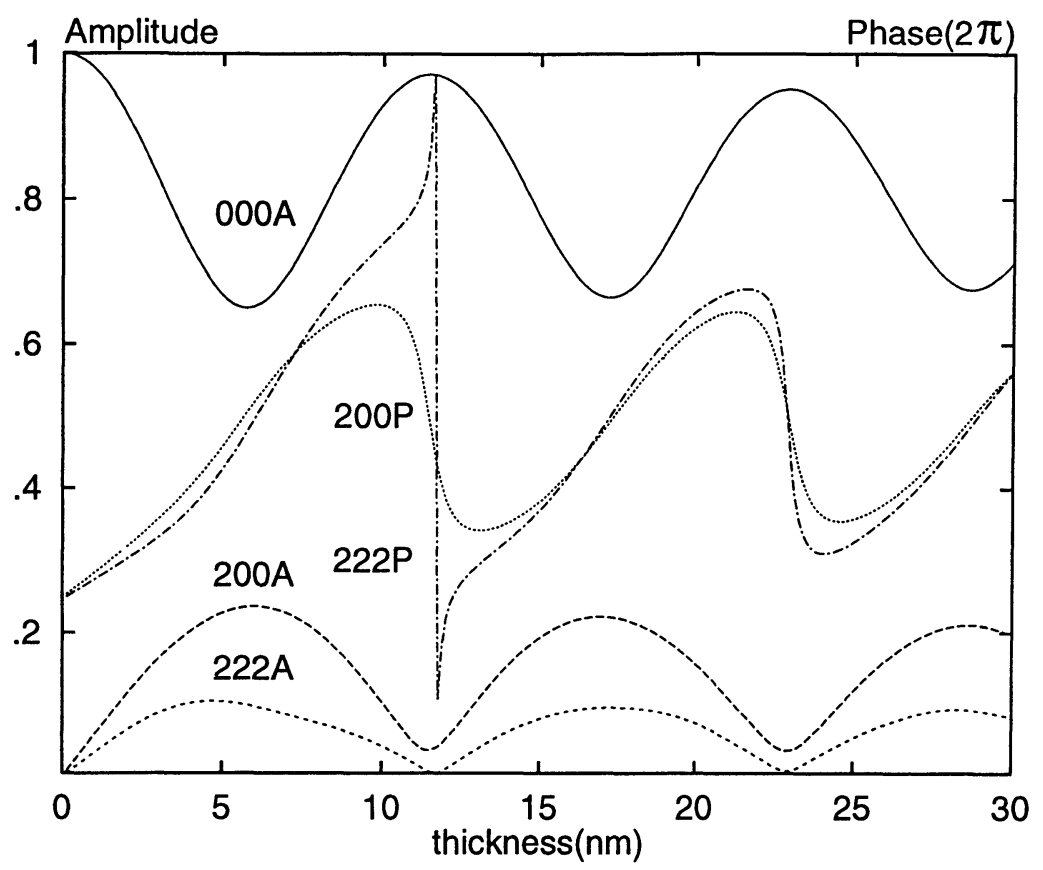

a)

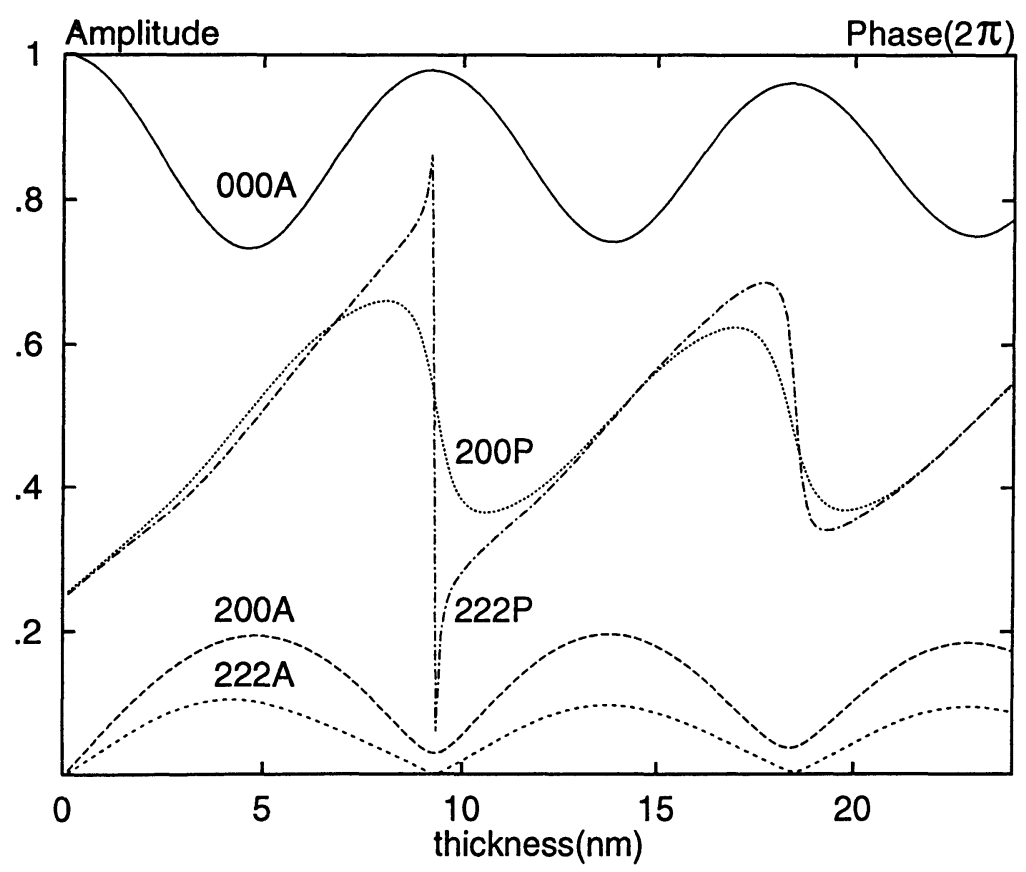

b)

Fig. 6. - The amplitudes $(A)$ and phases $(P)$ of beams (000), (200) and (222) with different "approximation" and "iteration" conditions. The phases are relative to the central beam so that the phase of $(000)$ is not shown. To make the figures clearer, the used unit of phase is $2 \pi$. Figures $6 \mathrm{a}, 6 \mathrm{~b}, 6 \mathrm{c}$ and $6 \mathrm{~d}$ are obtained by tactics \#5, \#6, \#7 and \#8 respectively as in Figures 5 and 1 . Note the different $x$-scales for different figures. 


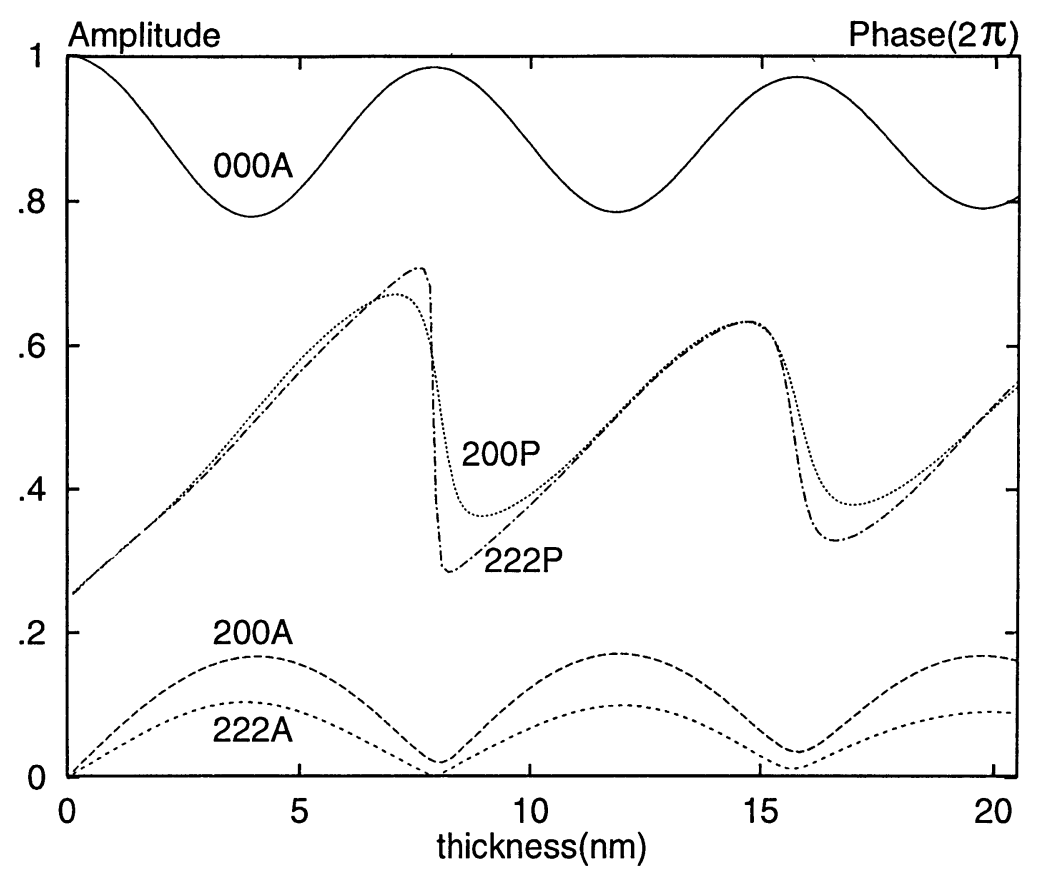

c)

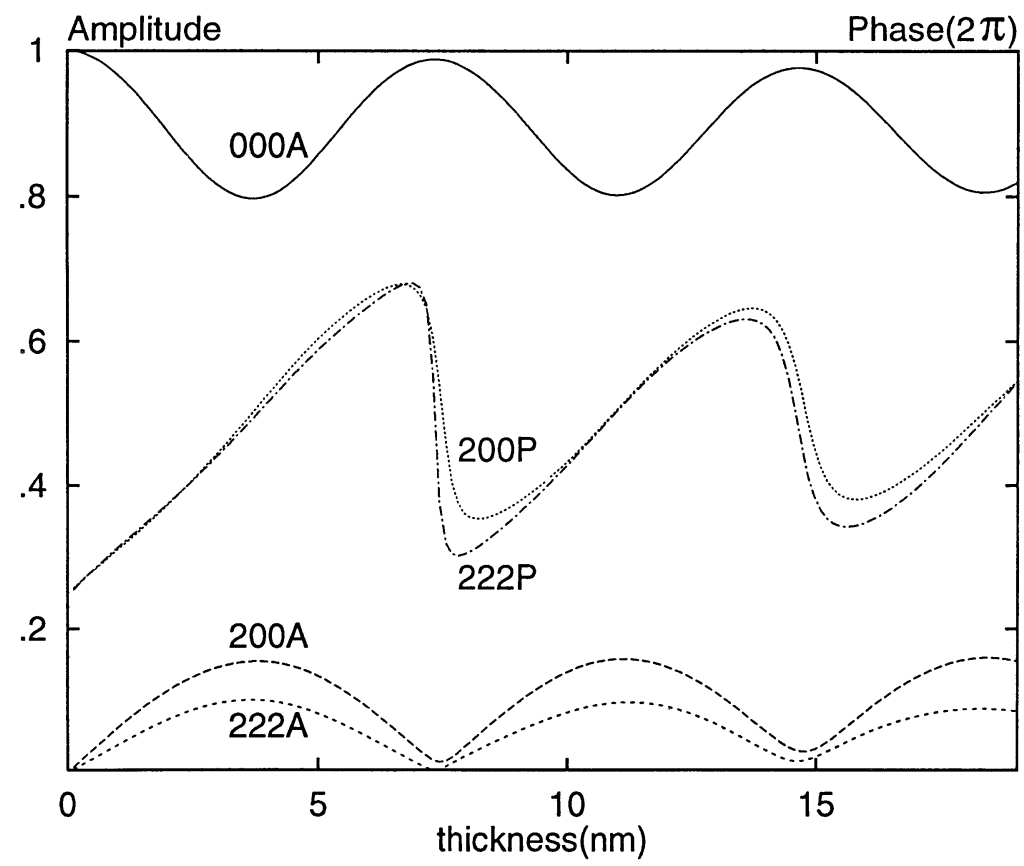

d)

Fig. 6. - (continued) 
Figure 5 shows the Pendellösung curves, with a reasonably small damping, corresponding to the tactics \#8, \#7, \#6 and \#5 shown in Figure 1, with $\Delta z=c / 4,(M \times N)=(64 \times 64),(64 \times$ $32),(32 \times 32)$ and $(32 \times 16)$, and $\rho_{\min }=2.3,3.7,6$ and $9 \mathrm{pm}$, respectively. It should be noted that, according to the above discussions, they all represent valid results, although they show very different thickness periods $P$. The normalization $I_{\text {dif }}$ factors show no dramatic little differences, as those given in the last section. The curves with better "approximation", because of smaller phase-shift-change $\Delta \chi_{\max }$, have the worse "iteration", because of smaller $(M \times N)$ size, and vice versa.

Since they are all "good" calculations, we should believe all of them. How can this be, since these very different curves surely do not give identical results. However, if we replot these Pendellösung curves with a different thickness scale, together with the curves concerning some other main diffracted beams, as shown in Figure 6, it is interesting that they have nearly identical shapes and relations. The curve for beam (111), which nearly coincides with the curve for beam (200), is not plotted here for simplicity, but instead the beam (222) is drawn. All the beams with nonnegligible intensity have nearly identical behaviour and similar period $P$.

It is reasonable to suppose that the simulated images at the corresponding thicknesses will be similar since the exit-waves are similar. This is confirmed in Figure 7, where the first rows of simulated images in both parts (a) and (b) are the results concerning curve \#5 in Figure 5, the second rows are those concerning curve \#6, and so on. The Figure 7a corresponds to a thickness $T$ for which the beam $(000)$ has its second minimum value, and the Figure $7 \mathrm{~b}$ corresponds to $T$ between this second minimum and the third maximum. The corresponding simulations are very similar to each other, except for (b) at the defocii $\Delta f=-70 \mathrm{~nm}$ and $90 \mathrm{~nm}$ where a slight contrast difference in the centre of the black dots can be observed.

Until we have found another way of knowing which of these four tactics is the best, we must accept the fact that all these simulations are valid in the view of simulation techniques. There is thus a problem of knowing exactly the real value of $T$ according to the image simulation. This is not a new problem. As long as there is a periodicity $P$ for the Pendellösung curves, the simulated images will repeat with $P$, so that the real thickness $T$ could be the thickness according to image matching, $T_{\text {ima }}$, plus or minus an integer multiple of the depth period,

$$
T=T_{\text {ima }} \pm n P \text {. }
$$

A serious problem concerning the image similarity is that if the corresponding $T$ is chosen at the peak position of the transmitted beam, the similarity between images obtained using different tactics could be destroyed. Figure 8 shows an example where the defocus $\Delta f$ is $-110 \mathrm{~nm}$ and the thicknesses, from left to right, are $T=11.50,9.20,7.92$ and $7.28 \mathrm{~nm}$, corresponding to the second peak of the beam (000) in Figure 6. However, if we use a normalized contrast factor for an image defined as

$$
C=\left(1-I_{\min } / I_{\max }\right),
$$

$I_{\max }$ and $I_{\min }$ being the maximum and minimum pixel intensities in the image, it is found that all the images calculated with the thicknesses used in Figure 8 have very low contrast if we compare them with the contrast of the images calculated for other thicknesses, such as those used in Figure 7. When the difference in image feature is recognizable, the maximum contrast at the thickness corresponding to the second and third maximum for beam $(000)$ is as low as $C_{\max }=10.0 \%$, compared with the minimum contrast observed in Figure 7, $C_{\min }=40.0 \%$, and compared to the averaged contrast of all 32 images shown in Figure $7,\langle C\rangle=60.6 \%$. With such low contrast, it is very difficult to observe any feature in the experiment, so that the conclusion for the thickness uncertainty (8) will not be violated.

In this connection, the reason why the first two images shown in Figure 8 display an abnormal appearance, although the $\mathrm{Cu}[110]$ symmetry is not destroyed, is not clear and is being studied. 


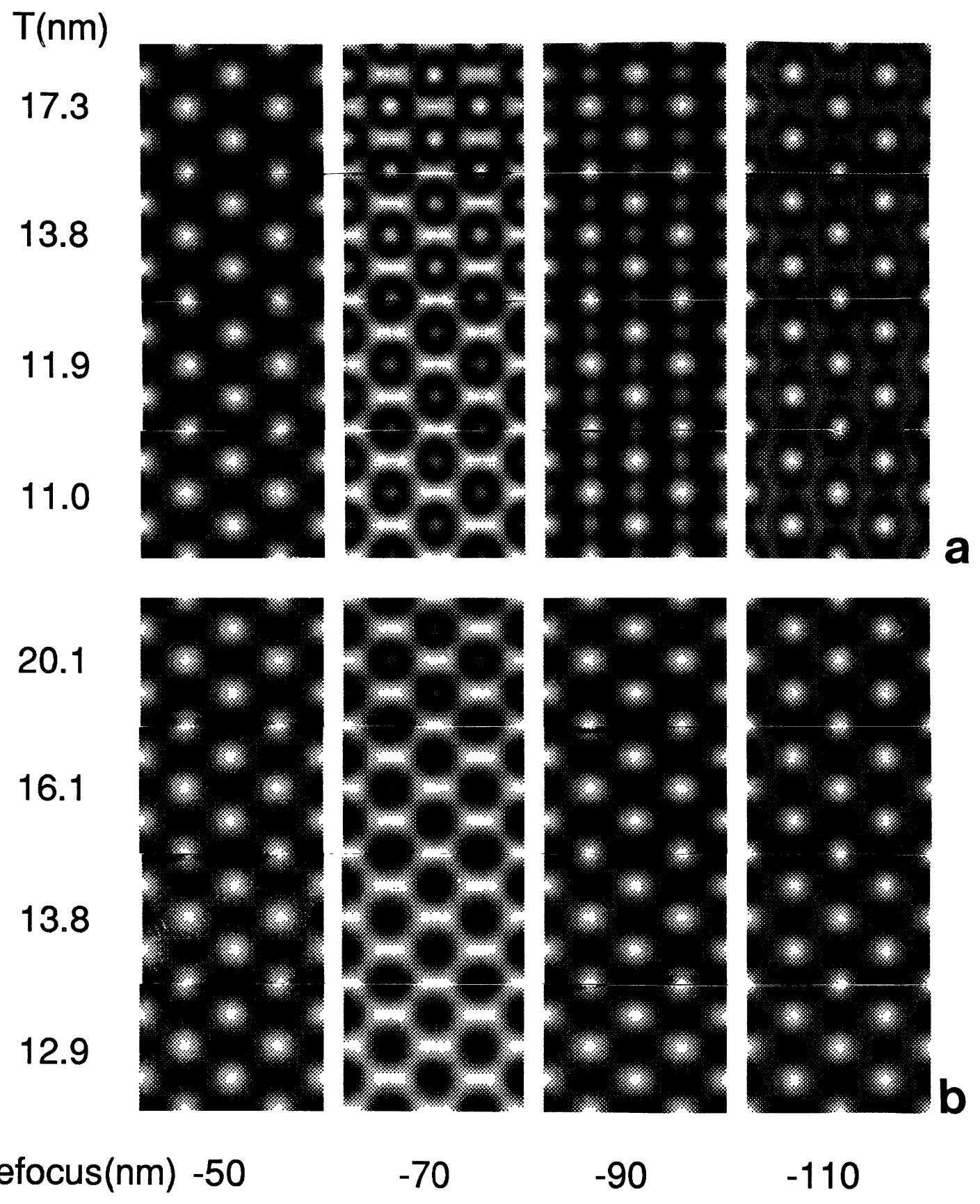

Fig. 7. - The simulated copper $\langle 011\rangle$ images for all four "valid tactics" \#5, \#6, \#7 and \#8 shown in Figure 6. The crystal thicknesses $T$ are chosen such that they locate at the second minimum of beam (000) (for $7 \mathrm{a}$ ) and at the middle between this minimum and the third maximum (for $7 \mathrm{~b}$ ). The resemblance is quite good. 

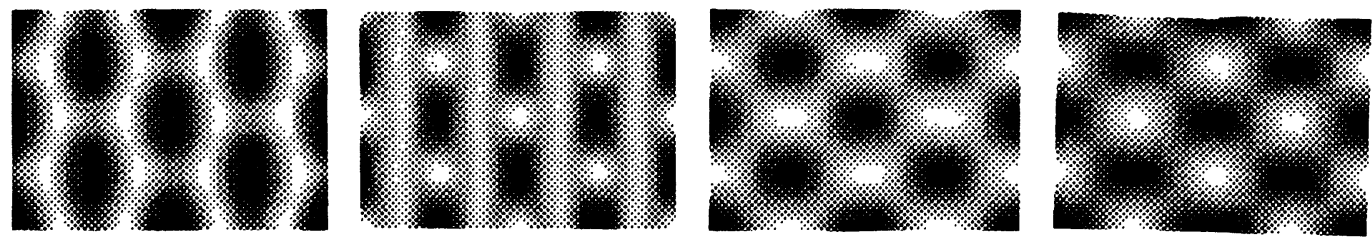

Fig. 8. - Simulated images at the thicknesses corresponding to the second maximum of beam (000) in Figure 6: $T=11.50,9.20,7.92$ and $7.28 \mathrm{~nm}$ respectively, from left to right. They correspond to the worst similarity. A microscope defocus of $\Delta f=-110 \mathrm{~nm}$ is used. The contrast factors are $C=8.4 \%, 2.8 \%, 6.8 \%$ and $6.1 \%$ respectively.

This effect cannot result from numerical errors which could have been introduced by the subslicing of the unit cell, since all the simulations shown in Figure 7 and the two others shown in Figure 8 are all sub-sliced in an identical way $(c / 4)$. It probably arises for particular combinations of the (defocus/thickness) values when a strong central beam is dominant.

It is not surprising that the images at the thickness corresponding to the maximum intensity of the transmitted beam have very low contrast. Since, according to the Abbe principle, an image is the result of the interference of all the beams, the interference between the very strong transmitted beam and several very weak diffracted beams creates an image with very low contrast. That can be shown, from (2), by an ideal phase object expansion

$$
\exp (i \chi) \approx 1+i \chi+\left(\chi^{2} / 2\right)+\ldots
$$

where any other terms are too smaller than unity.

It also needs to be stressed that the fact that similar images are obtained for different thicknesses with different tactics is not dependent on having periodic Pendellösung curves, as long as these have similar shape but different thickness scale.

\section{Discussion}

In Section 2, the effects caused by different minimum impact parameters $\rho_{\min }$, maximum projected potentials $\phi_{\max }^{\mathrm{P}}$, slice thicknesses $\Delta z$ and Fourier space sizes $(M \times N)$ have been studied one by one, individually. It should be noted that in the conventional method the value of $\phi_{\max }^{\mathrm{P}}$ is not independent of the choice of the other parameters, but depends on $(M \times N)$, which defines the limit of the sum of the Fourier coefficients

$$
\phi_{\max }^{\mathrm{P}}=\phi^{\mathrm{P}}(0)=\sum_{h, k} \tilde{\phi}_{h k 0}
$$

$h$ and $k$ varying from $(-M / 2)$ to $(+M / 2)-1$ and from $(-N / 2)$ to $(+N / 2)-1$, respectively, while the atomic chain in question is at the origin. Therefore in the conventional method the effects of $(M \times N)$ and of $\phi_{\max }^{\mathrm{P}}$ cannot be separated.

A satisfactory interpretation of the Pendellösung curve periodicity $P$ in the many-beam situation has not been given previously, except for the simple two-beam case $[12,13]$. A full understanding of this periodicity is important for choosing the best simulation conditions, or tactics as used above, and for reducing the thickness range in simulation. If we approach it in a very simple way, that is if we regard the specimen as an ideal phase object and the projected potential as an 
averaged value $\left\langle\phi^{\mathrm{P}}\right\rangle$, the averaged exit-wave will be periodic with periodicity

$$
P \approx \frac{2 \pi}{\sigma\left\langle\phi^{\mathrm{P}}\right\rangle} .
$$

All the beams in reciprocal space, which represent the 2D Fourier transform components of the exit-wave, should have a similar period in the $z$ dimension. This simple assumption can, of course, predict only qualitatively the behaviour of $P$. There are five different conditions for changing $P$ : (i) when $\rho_{\min }$ decreases, $\phi^{\mathrm{P}}$ increases at the centre of an atomic chain but remains unchanged for all other pixels, so that $\left\langle\phi^{\mathrm{P}}\right\rangle$ increases and, according to (12), $P$ decreases (see Fig. 2); (ii) when $\Delta z$ decreases, $\phi^{\mathrm{P}}$ does not change as long as $\Delta z$ includes an integer number of halfatoms, so that $\left\langle\phi^{\mathrm{P}}\right\rangle$ and also $P$ remain constant (see Fig. 3); (iii) when $(M \times N)$ increases, this creates extra pixels between each two original pixels: owing to the non-linearity of the potential, the averaged value of any two new pixels adjacent to an original pixel will be greater than the value corresponding to the original pixel, so that $\left\langle\phi^{\mathrm{P}}\right\rangle$ increases slightly and $P$ decreases slightly (see Fig. 4); (iv) when $\rho_{\min }$ decreases and at the same time $(M \times N)$ increases, the increase of $\left\langle\phi^{\mathrm{P}}\right\rangle$ is more prominent, so that $P$ decreases more than when only one of them is changed (see Fig. 5); (v) when the accelerating voltage decreases, the interaction constant $\sigma$ increases and so, again according to (12), $P$ also decreases. This last point is confirmed by computer simulation and also demonstrated by Glaisher and Spargo [14] (although diamond at $100 \mathrm{kV}$ is an exception: this exception could be a consequence of some different simulation conditions, size and $\Delta z$, etc..., because no computing conditions were given there).

It must be noted that this phase-object explanation (12) of the Pendellösung period $P$ is far toc simple. Although it does predict the tendency of the period change, a quantitative analysis is completely out of the question. An extinction distance $\xi$ can also be used as a guide to the period $P$ (see for instance [12] p. 102)

$$
\xi=\frac{\pi}{\sigma \tilde{\phi}_{000}}
$$

which leads to the same conclusion as (12).

In the conventional method, when $(M \times N)$ is big enough, there is always a $P$ limit. This means that beyond a certain $(M \times N)$ size, a further increase will not produce noticeably different Pendellösung curves. In Figure 9 the results for conventional calculations with $\Delta z=c / 4$ and different $(M \times N)$ sizes are shown, which are the counterparts of the direct calculations shown in Figure 5.

Although the $(M \times N)$ size convergence is found more easily with the conventional method than with the direct one, the former is physically not as precise as the latter because of the high $\left\langle\phi^{\mathrm{P}}\right\rangle$ value (11) violating the validity criterion (3 or 6$)$ of the quasi-classical approximation. This is also reflected by the facts (i) that the damping effects on the conventional curves appear more severe than on the direct ones, and (ii) that the $I_{\text {dif }}$ factors values are lower: $0.85,0.83,0.79$ and 0.80 for $T=30 \mathrm{~nm}$, to be compared with the values $0.93,0.91,0.90$ and 0.89 obtained using the direct method. The better convergence shown by the conventional method is the result of the extra high value of $\phi^{\mathrm{P}}$ at the centre. For example, for curve \#5 in Figure 5, if $\rho_{\min }$ is reduced from 9 to $4 \mathrm{pm}$, the new curve will have a $P$-value similar to that for curve \#6, but at the same time $\Delta \chi_{\max }$ will increase from 0.036 to $0.089 \mathrm{rad}$ and $I_{\text {dif }}$ will decrease from 0.89 to only 0.80 .

At large $(M \times N)$ sizes and for both methods, $\left\langle\phi^{\mathrm{P}}\right\rangle$ tends to be constant, because the $\phi^{\mathrm{P}}$ distribution is approximately linear for small $\rho$ values. For very large $(M \times N)$ sizes, $\left\langle\phi^{\mathrm{P}}\right\rangle$ will be dominated by only a small $\rho$ value, for which $\phi^{\mathrm{P}}$ is much bigger. The averaged value of any two additional pixels on both sides of one original pixel will therefore be nearly equal to its value and 


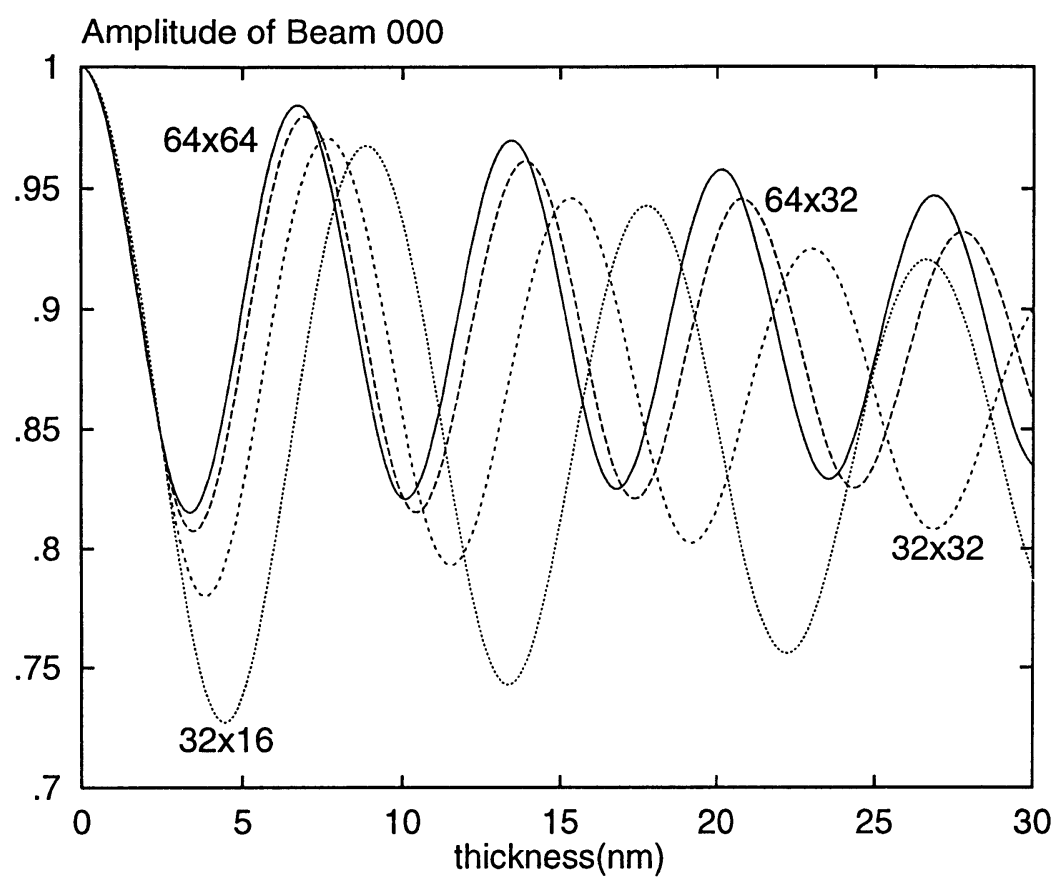

Fig. 9. - The Pendellösung curves obtained by the conventional method with $\Delta z=c / 4$ and with different $(M \times N)$ sizes, as indicated in the figure. They should be the counterparts of the curves shown in Figure 5 for the direct method.

the Pendellösung curves will converge. Since the conventional method uses a relatively higher central projected potential or a smaller $\rho_{\min }$ than the direct method, it converges for relatively smaller $(M \times N)$ sizes.

\section{Conclusion}

The main advantage of the direct potential method in multislice HREM image simulation is that it ensures the validity of the calculated transmission function.

According to the above analysis, the proper procedure is that outlined below: (i) the acceptable maximum value of the phase-shift-change over a distance of the order of the incident electron wavelength, $\Delta \chi_{\max }$, is chosen; (ii) the projected potential at the centre of an atomic chain, $\phi_{\max }^{\mathrm{P}}$, is determined by choosing a suitable value of $\rho_{\min }$ according to the sampling distances $\Delta x$ and $\Delta y$, or the $M$ and $N$ numbers, so that the calculation represents the real projected potential; (iii) the calculated Pendellösung curves are tested: if the damping effect appears to be too severe or the normalization $I_{\text {dif }}$ factor too small for the studied thickness $T$, either the slice thickness $\Delta z$ is reduced or the $\rho_{\text {min }}$ is increased slightly; (iv) the $(M \times N)$ size is increased in order to obtain nearly unchanged Pendellösung curves when $(M \times N)$ is increased further, if one pursues a "reliability" strategy similar to that adopted in the conventional method.

The period $P$ of the Pendellösung curves in the many-beam condition can be estimated qualitatively by a simple consideration of an ideal phase-object with averaged projected potential, which helps us to analyse and compare these curves. The thickness uncertainty should also be taken into consideration. 


\section{References}

[1] Tang D. and Dorignac D., Proc. ICEM-13- PARIS (Les Éditions de Physique, 1994) pp. 377-378.

[2] Tang D. and Dorignac D., J. Microsc. 179 (1995) 191-200.

[3] Carlson T.A., Lu T.T., Tucker T.C., Nestor C.W. and Malik F.B., Oak Ridge National Laboratory report (1970) ORNL-4614, USA.

[4] Glauber R.J., Lectures in Theoretical Physics, Vol. I (Interscience, New York, 1959) pp. 315-414.

[5] Doyle P.A. and Turner P.S., Acta Crystallogr. A24 (1968) 390-397.

[6] Peng L.-M. and Cowley J.M., Acta Crystallogr. A44 (1988) 1-5.

[7] Tang D. and Dorignac D., Acta Crystallogr. A50 (1994) 45-52.

[8] Kilaas R., O’Keefe M.A. and Krishnan K.M., Ultramicroscopy 21 (1987) 47-61.

[9] Self P.G., O’Keefe M.A., Buseck P.R. and Spargo A.E.C., Ultramicroscopy 11 (1983) 35-52.

[10] Cowley J.M. and Moodie A.F., Acta Crystallogr. 10 (1957) 609-619.

[11] Self P.G. and O'Keefe M.A., HRTEM and Associated Techniques (Oxford Univ. Press, New-York, 1988) pp. 244-307.

[12] Hirsch P., Howie A., Nicholson R.B., Pashley D.W. and Whelan M.J., Electron microscopy of thin crystals (Krieger Pub. Co., London 1977) pp. 201-205.

[13] Spence J.C.H., Experimental High Resolution Electron Microscopy, 2nd Ed. (Oxford University Press, New York, 1988), pp. 160-161.

[14] Glaisher R.W. and Spargo A.E.C., Ultramicroscopy 18 (1985) 323-334.

[15] Stadelmann P.A., Ultramicroscopy 21 (1987) 131-146 and http : //cimewww.epfl.ch : 80/EMS/EMS. html (1995). 